\title{
Explore the Visual Language of Costume Design
}

\author{
Linshan Zhang \\ Jiangxi Institute of Fashion Technology, Nanchang, 330000, China
}

\begin{abstract}
Keywords: Costume design, Visual Language, Material element, Geometry elements
\end{abstract}
\begin{abstract}
The clothes designers need to comprehensive analysis to consider the connection between the various factors, from the market, sales, clothing basic visual language, history and trends, need to grasp and analysis and design on whole garment basic properties and characteristics of the guarantee can effectively convey visual language, establishment conforms to the national development of clothing brand, promote national economic development, this paper mainly introduces the basic characteristics of visual language and concepts, involved in the process of analysis in the clothing design color element, geometric elements and material elements, fully analyzing the basic relationship between visual art and clothing design.
\end{abstract}

\section{Introduction}

One of the most important human intuition is visual, we can intuitive cognition of things use eyes, the visual arts is to summarize and analyze the people can actually see the visual image of the experience and feeling, to find the rule of design aesthetics, which can be formed, the new visual image, of beauty in real life, can exist everywhere in visual art, because the visual art has different forms and scope, but all kinds of visual arts will be affected by the visual language and guidance, based on the theory of visual art as the basic application, the general expression of visual arts is called visual language symbols. In the clothing design process, based on visual language to design the clothing visual effect, analysis the particularity of design clothing, so that to the potential of deep excavation design clothing, in the process of actual operation and design, visual language has a certain effect, on the basis of the analysis and comparison of visual language, is an effective way of design of clothing, visual language or design clothing are important inspiration.

\section{Overview and characteristics of visual language}

Visual language mainly includes two parts, basic elements and principles of visual design; it must convey the significance to form a composite system. Thinking on the organization design basic principle is actually need to pass and artistic conception, mainly includes: contrast, layout, unity, balance; Basic elements of the main means in the design of the most basic elements, like the designer's computer, the artist's pen, is the basic foundation of design, including shape, line, texture, space, light and shade, etc. Visual language is a kind of strong applied discipline, main purpose is to develop the market, not only the designers and artists of noun, involved in the design process of material value and spiritual significance, are closely related with basic needs, people involved in social economic basic functions and departments. As growing grassroots culture and international fashion, the spread of culture, science and technology progress, make visual language is more than just a simple art symbols, visual things are the first contact information of the organs, become more important in the design process, receive more attention. Visual language is more than just the carrier of information, also has many advantages in the expression of emotion, can on the emotion and image level, have quite a lot of advantages. Production as a result, people in real life, the visual language has the rich connotation of culture, at the time of relay sensory information, can express more elements deep emotional development, further to attract people to understand and know. At the time of express and convey cultural language, there is a big obstacle which can reasonable use other kinds of ways to express the unspeakable emotion incisively and vividly, find visual far has certain practicability, and have been able to transcend literary language, it is because of this that can use their appreciation and reading way to express the different culture, different countries, different class, different languages, 
different nationalities, different color of skin on the work, has the following three characteristics: including the transformability and can pass the objectivity and universality, participatory and timeliness $^{[1]}$.

\section{Visual element and its significance in the design of clothing}

\section{Geometrical element}

Arts and crafts, in fact, including clothing design, so the design of clothing involved in the process of the basic elements of basic composition elements of the design of similar to the arts and crafts, have many similarities, and in the process of design, reasonable use of elements, the need to comply with certain rules is similar or the same. In the design of costume style, mainly is the design of clothing color, shape, fabric, etc., design the basic visual elements is also include clothing color, texture, materials and dot, line, face, body, but in the actual design process, there is a lot different specific space and material, and the specific results, and there are some special place on the form. Basic composition elements of the design of point, line, face, because it will be affected by fault, visual habit and so on, different ways to arrange the time point, line and plane, which can form different visual effects. Have a point in a plane, it will focus on vision. In the plane distribution around the two points, can form a certain balance. Two oblique distribution points, it will get a dynamic visual. The vertical line leading to visual feeling of tall, slender; To lateral horizontal extension and feel quiet; Linear softness, soft arc. If you can put the above elements to the change of the size, thickness, shape, it can also lead to more visual effects. At the time of design clothing, constitute the basic elements, also has the characteristics such as dot, line, face, but if it is in a specific environment design clothing, often by certain restrictions, and form a special visual effect.

At the time of design clothing, some elements mainly reflects in pockets, collars, belts, card, brooches, buttons and other relatively small parts; At the time of design clothing, line elements mainly reflects in the garment place the front line, line, decorative lines, line, contour, etc.; In the process of design clothing, surface elements mainly reflects in the garment body before and after the big patch pocket, joining together face, clothing, etc. In clothing design, dot, line, surface specific performance can be found that at the time of design, not only need a few basic elements, also need to have a certain practical function and the concrete structure of the need to conform to the dress designing stereo effect ${ }^{[2]}$.For example, in designing clothes pocket when application point element, not simply the basic forms of art to design the pocket size, shape, need to use the method to determine, design pocket, need to give full consideration to use pocket shape, size, etc. To conform to the actual functional requirements, using a pocket is convenient, such as at the time of design in bag depth needs to be higher than the hand length, etc. Only ensure harmonious and unified visual effect and the function, but to keep clothes not only has a certain aesthetic feeling, also very practical, but also not to say that, at the time of design basic composition elements of limited function and structure, make it lose the original visual design effect, can't achieve the goal of flexible and varied. In fact, in the design, the most important thing is the basic composition element such as dot, line, face, is also an important factor in design of rich and colorful clothing. Design clothing, in the process of point, line and plane combination and change, for example, pocket shape and position, button arrangement and location, the shape of the front line and line etc., which are all express design dresses are the important factors of visual language.

\section{The elements of color}

The strongest language is the most abundant and color, all the design of the visual effect is closely related to color, fashion design but also pay more attention to the color. If use different color of the fabric to design the same style, can form different design feeling. People have various ideas, each color express different feelings, with appropriate symbolic, and there will be a different psychological Brahman. For example, white represents quiet and pure; Red stands for excitement, passion, joy; Green represents peace, life; On behalf of the elegant, deep blue, etc., the different color with different degree of purity and lightness, can form more colorful visual effects, it is color is important part of the visual language. In clothing design, the choice of clothing color process, need to use to determine the overall effect of color, color can be in a certain extent, emphasize and foil costume 
design visual effects, color value existence and visual language is not just a single color, need reasonable contrast two or more colors, for example, compare different color fastens with color, brightness and purity, by comparing can improve the impact of the color to a certain extent, enrich the visual language ${ }^{[3]}$.

\section{Material elements}

In the process of design of clothing, in the visual arts, graphic composition, color composition style color belong to the content of the performance under different forms, such as the material is actually design clothing, it needs to have a special language reflected, clothing design using the main material is compared with visual factors to the textile material, has a special performance, more fluid, some textiles has certain elasticity, plasticity and drapability, from the textile texture, form the visual effect of the factors, other material is incomparable. Different skin texture and materials are not cause different visual effect, for example, represents fine delicate, exquisite; Rough representative vibration straightforward, atmosphere; Light represents elegant, soft; Osteoporosis represent the casual and comfortable; Flash with luxuriant, avant-garde; Smooth representing the exquisite, elegant, and so on, therefore, design of clothing materials not only influence the overall design effect, it is the key difference between design. In a costume, if comparing different history, different materials of fabrics, there will be a different situation of contrast. The material of clothing design, color and style have their own rich meaning, expressing the characteristics of different ${ }^{[4]}$.In the design of clothing, the above three aspects of the same signs are actually make it to be more rich visual language. But in every design clothing, need around certain content or theme, so, in the concrete use of visual language, it needs to be focused and have a choice, the organic combination of the above three factors, make sure you are able to achieve the goal of perfect design. The point of the costume design is to design the visual center, according to the different color or contrast of color to express the quality of a material is qualitative, outstanding point, in general, this expression is mainly applied to the process of design pockets, buttons. According to different quality of a material, color, texture, etc. all of them are needed to show the shape of the clothing design of plane and line.

\section{The relationship between the visual language design and other visual design in the clothing design}

After long time development, the clothing visual design art and other visual design art supplement each other all the time. Development history, analysis and research on the east and the west can be found, which tend to have decoration, architecture, painting, and many other art forms, the decoration art form is an important creative dynamics design clothing, in addition, for other visual design, apparel visual language to provide a certain amount of inspiration. Other visual art and clothing through the visual language contact between the visual arts, in addition, in the same historical stage, the different forms can react in a certain extent, the same content, such as at the time of the Renaissance, in conformity with the wind to wide and padded clothing construction; the characteristics of the Los. Modern clothing in the process of design, need to meet all kinds of visual effects, don't report the loss of processing details, or integral style, to be able to reflect the apparel design will be affected by the visual design. From the design on the garment terminology it can be found, for example, design clothing building wind, sculpture, wind and surrealism, etc., although there are many parts of the visual language design dress is similar to other visual art design, but in the actual design process, it will be affected by the space shape, for example, the limitation of the human body movement and comfort, the human body, at the time of design clothing, need reasonable adjustment related to visual art, so as to make sure you are able to redesign, make sure you are able to show costumes feature in the effective space. For example, Yves saint Laurent in the process of design, main is to use abstract Mondrian's cold, not intercept partial image, but need to catch cold abstract essence, prompted in special space, cold clothing design can not only express the inner abstract effect, it can fully meet the visual effect.

In the process of design clothing, compared to other design, visual design and visual language, one of the most obvious is the material difference, the diversity of material makes has unique advantages in the design of clothing, in the design of graphic poster, visual language use in graphics set color, 
there will be no changes due to the clothing materials form the unique effect. Ceramics, building has stationary and solidification of beauty; Costume design in fact human activity can be used to express the change of fabric and flow of the United States, it also officially because of this, which makes the design clothing, the visual language is different from previous visual design art, with different expression, contrast fabric material, stitching, hand-painted, overlap, etc., to fashion design which have a certain goal, promote the development of clothing design industry ${ }^{[5]}$.

\section{Conclusion}

Above all, costume design, painting, architecture, decoration and so on all are visual art, more attention to decorative, because in the design process is designed, with differences between the elements used to pay more attention to the importance and characteristics of art design, and design ideas of the freely crisscross, fashion trends, such as making clothing, it has more sensitive visual language, and more and more be taken seriously. In the process of design clothing, reasonable to join the visual language, it is more convenient for comparing with other art, on the analysis of the effect of performance, technique of expression, it not only needs to improve the design concept of clothing, but also needs to expand the design character of thought and view, promote the development of clothing design.

\section{References}

[1] Zhang Jiaqiu, Liang Huiying. Beijing Opera for the application of visual elements in knitting clothing design. Journal of textile guides,2012(7):70-72.

[2] Chen Xianjin. Theory of costume design in the visual language of tension and its penetration. Journal of Taiyuan city vocational and technical college , 2010(8):155-156.

[3] Zhao Siyu. Combining fashion clothing design discusses the design principles and reflect. Science and technology entrepreneurs,2013(8):240-240.

[4] Tan Juan. Romanticism in clothing design. Journal of literary life, literature and art theory,2013(1):72.

[5] Zeng Yundong. Mark applications in the field of contemporary fashion design. Journal of beauty and age (first half),2010(4):35-39. 